\title{
Planar Voronoi cells: the violation of Aboav's law explained
}

\author{
H. J. Hilhorst \\ Laboratoire de Physique Théorique, Bâtiment 210, Université de Paris-Sud \\ 91405 Orsay Cedex, France
}

April 21, 2018

\begin{abstract}
In planar cellular systems $m_{n}$ denotes the average sidedness of a cell neighboring an $n$-sided cell. Aboav's empirical law states that $n m_{n}$ is linear in $n$. A downward curvature is nevertheless observed in the numerical $n m_{n}$ data of the Random Voronoi Froth. The exact large- $n$ expansion of $m_{n}$ obtained in the present work, viz. $m_{n}=4+3(\pi / n)^{\frac{1}{2}}+$ $\ldots$, explains this curvature. Its inverse square root dependence on $n$ sets a new theoretical paradigm. Similar curved behavior may be expected, and must indeed be looked for, in experimental data of sufficiently high resolution. We argue that it occurs, in particular, in diffusion-limited colloidal aggregation on the basis of recent simulation data due to Fernández-Toledano et al. [Phys. Rev. E 71, 041401 (2005)] and earlier experimental results by Earnshaw and Robinson [Phys. Rev. Lett. 72, 3682 (1994)].
\end{abstract}

LPT - ORSAY 05/89

${ }^{1}$ Laboratoire associé au Centre National de la Recherche Scientifique - UMR 8627 


\section{Introduction}

\subsection{General}

In nature, planar cellular systems come in a wide variety. They include biological tissues 1, 2, 3, polycrystals 4, cells formed by particles trapped at a water/air interface [5], cells in surface-tension driven Bénard convection [6], in two-dimensional soap froths [7], and in magnetic liquid froths [8]. In other systems cellular structure may appear when the data are subjected to the Voronoi construction 9]. Examples of these are hard disks on an air table [10, a binary liquid during late stage coarsening [11, two-dimensional colloidal aggregation [12, 13, 14, nanostructured cellular layers [15], and studies of two-dimensional melting [16].

Two empirical rules play a prominent role in studies of planar cellular systems: Lewis' law and Aboav's law. Both are statements about the statistics of a cell's most conspicuous properties, viz. its area and its number of sides. Lewis' law [1] says that the average area $A_{n}$ of an $n$-sided cell increases with $n$ as

$$
A_{n}=\frac{a_{0}}{\lambda}\left(n-n_{0}\right),
$$

where $a_{0}$ and $n_{0}$ are constants and $\lambda$ is the areal cell density. In the present work we are interested in the second one of these laws, formulated by Aboav 4], who noticed that many-sided cells tend to have few-sided neighbors and vice versa. He expressed this correlation in terms of the average $m_{n}$ of the number of sides of a cell that neighbors an $n$-sided cell. Aboav's law, also called the Aboav-Weaire [17] law, asserts that

$$
m_{n}=6-a+\frac{b}{n}
$$

where $a$ and $b$ are numerical constants. This law, which expresses an intuitively plausible trend with $n$, is in widespread use 2, 3, 5, 6, 7, 8, 10, 11, 12, 15, 16] in the analysis of experimental data on cellular structures. One usually plots $n m_{n}=(6-a) n+b$ as a function of $n$ and often refers to either this relationship or equation (1.2) as the linear law.

The surprising fact is that for many of these cellular systems, in spite of all their diversity, Aboav's linear law appears to hold with good accuracy in most of the experimentally accessible range, which runs from $n=3$ to $n$ typically between 9 and 12. The experimental values of the Aboav parameters, listed e.g. in reference [18, are typically in the range $0.7 \lesssim a \lesssim 1.5$ and $5.7 \lesssim$ $b \lesssim 8.5$ [19. Attempts to explain the linearity with $n$ and the numerical values of $a$ and $b$ from first principles, i.e., on the basis of a microscopic geometrical model, have been unsuccessful. The various derivations of (1.2) that one does find in the literature all involve approximations (usually of the mean-field kind; see e.g. reference [20]) or hypotheses whose general validity 
is subject to caution (we consider the 'maximum entropy method' 21] to be in this class). The dominant view today is probably that (1.2) is not exact, but merely a good approximation to some unknown 'true' curve, which need not be the same for all cellular systems.

It has been realized, in particular by Le Caër and Delannay [22], that knowledge about the large- $n$ behavior of $m_{n}$ will constrain the law for $m_{n}$, for example by establishing the regime of validity of its linearity or by putting limits on the numerical values of $a$ and $b$. The question of the large- $n$ behavior of $m_{n}$ is however a difficult one and has received little attention since. The 'derivations' referred to above generally lead to (1.2) without providing any restrictions on its range of validity. In the present work we return to the large- $n$ behavior. We analyze it within the context of the Random Voronoi Froth and consider the implications of our conclusion for the interpretation of simulations and experiments.

\subsection{The Random Voronoi Froth (RVF)}

We consider the 'Random Voronoi Froth' (RVF), in the mathematical literature rather called the 'Poisson-Voronoi tessellation'. It is obtained by constructing the Voronoi cells 9] of a planar set of randomly and uniformly distributed point centers, for convenience often called 'seeds' (but without the implication that they are material). The statistical properties of the RVF have been studied more than those of any other microscopic model of cellular structure. Reference 9] compiles a large body of analytic results and numerical tables. For the role and place of the RVF amidst other models of cellular systems one may consult, e.g., Rivier [23] or Schliecker [24].

Analytically, a first-principle derivation of an exact expression for $m_{n}$ in the RVF (or, for that matter, in any other geometrical model) is still lacking. The reason is that the calculation of this quantity is in the notoriously difficult class of many-body problems. Numerically, however, the $m_{n}$ values of the RVF are known with considerable precision [25, 26, 27, 28]. The best data come from Monte Carlo simulations by Brakke [28, whose results for $m_{n}$ have a four digit accuracy in the range $4 \leq n \leq 9$. In figure 1 we show these data as well as those by Boots and Murdoch [25], represented in the usual way in an $n m_{n}$ versus $n$ plot. The dashed straight line is Aboav's law (1.2) with $a=0.75$ and $b=5.76$. Although this law provides what appears as a very good approximation, the data points nevertheless exhibit an extremely small but distinctive downward curvature and the narrowness of their error bars rules out Aboav's linear fit. We have computed the second derivative $\left(n m_{n}\right)^{\prime \prime} \equiv(n-1) m_{n-1}-2 n m_{n}+(n+1) m_{n+1}$ from Brakke's [28] numerical data and plotted it in figure 1. The ratio $-\left(n m_{n}\right)^{\prime \prime} /\left(n m_{n}\right)$, which may be taken as a measure of the curvature, is of the order of only $1 / 250=0.004$. Hence a successful theory of the RVF should explain the existence as well as 
the sign and the smallness of the curvature. So far, in order to accommodate this departure from linearity, only ad hoc alternatives to (1.2) have been proposed (see, e.g., the discussions in [26] and in section 5.3.3 of [9]).

We pursue here the approach initiated in reference [29], where the methods of statistical mechanics were brought to bear on planar Voronoi tessellations. That work has opened the possibility of an expansion in powers of

$n^{-\frac{1}{2}}$ for all quantities of interest related to an $n$-sided Voronoi cell. This initially led to the asymptotic large- $n$ expansion of the probability $p_{n}$ for an arbitrarily chosen cell to have $n$ sides. An immediate further result was the proof that the RVF obeys Lewis' law (1.1) asymptotically for $n \rightarrow \infty$ with coefficient $a_{0}=\frac{1}{4}$. But whereas Lewis' law refers to a single cell, Aboav's deals with the intrinsically more difficult problem of cell-cell correlations; it therefore requires the separate study that we present here.

Our key result, equation (2.2) of section 2.1, is an exact expression for the asymptotic behavior of $m_{n}$ as $n$ becomes large. The inverse square root decay appearing in that expression explains the small deviations from linearity that are observed. As a corollary we obtain in section 2.2 the asymptotic expression for the total number, to be called $K_{n}^{(2)}$, of second-neighbor cells surrounding a central $n$-sided cell. In section 3 we discuss these results. We compare them to the numerical RVF data, as well as to data on diffusion limited colloidal aggregation coming from both experiments and simulations. Whereas our asymptotic expansion constitutes an exact result based on first principles, in section 4 we take a pragmatic attitude and try to find the best two- and three-parameter fits for $m_{n}$ that incorporate this large $n$ behavior. In section 5 we speculate briefly on modifications that our theory may undergo in the case of cellular systems not of the RVF type. In section 6 we conclude. Finally, in appendix A we prove a theorem concerning the statistics of randomly distributed points in a half-plane that is indispensible for the derivation of the main result.

\section{Two-cell correlations}

\section{$2.1 \quad$ First-neighbor correlation}

We will determine analytically the large- $n$ behavior of $m_{n}$ in a RVF. Two steps are required to arrive at this result. The first step of the derivation is based on the geometrical properties of the large $n$-sided cell; these have all been determined quantitatively in earlier work [29], where they required considerable mathematical effort. The arguments of the first step will be presented with the aid of a figure, but we stress that they are nonetheless nontrivial and dictated by compelling logic; they constitute the conceptually new part of this investigation. The second step is a problem in statistics whose solution, although somewhat lengthy, can be obtained by standard 


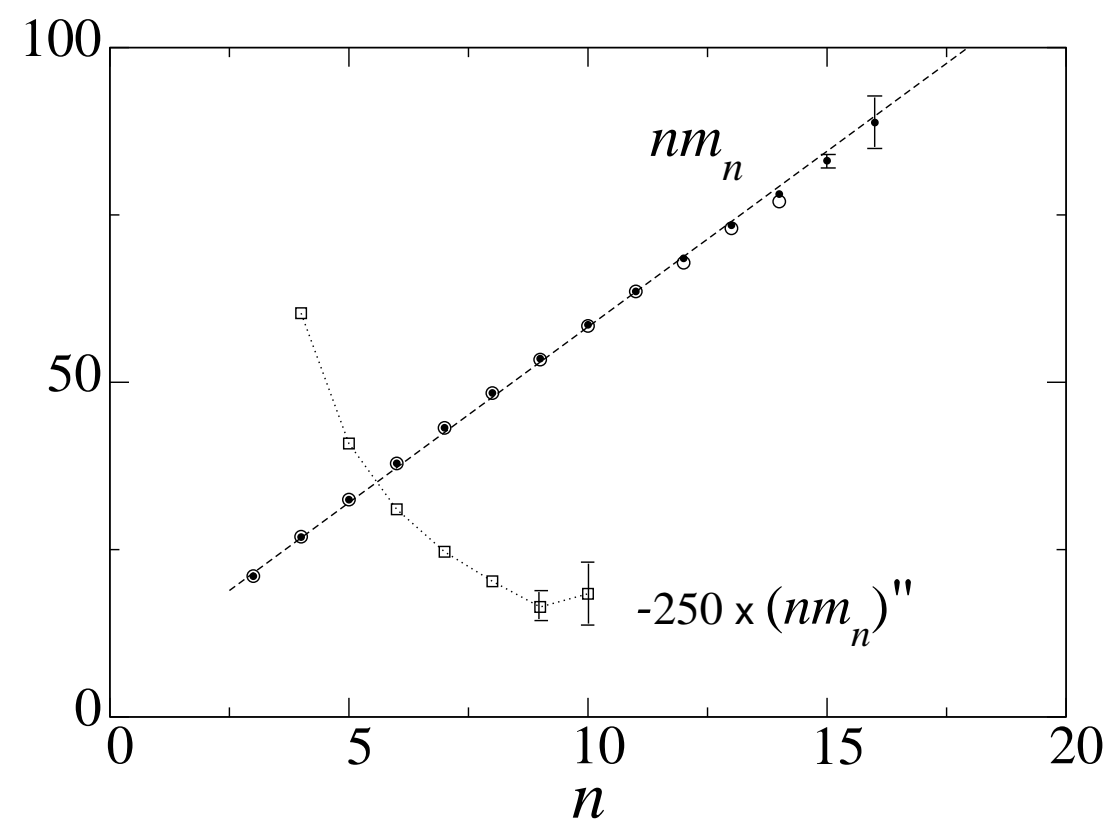

Figure 1: The cells neighboring an $n$-sided cell have together a total average of $n m_{n}$ sides. Open circles: simulation values of $n m_{n}$ according to Boots and Murdoch [25]. Dots: simulation values of $n m_{n}$ according to Brakke 28]. Dashed line: Aboav's linear law $n m_{n}=(6-a) n+b$ with $a=0.75$ and $b=5.76$. Open squares: the second derivative $\left(n m_{n}\right)^{\prime \prime} \equiv(n-1) m_{n-1}-2 n m_{n}+(n+1) m_{n+1}$, constructed from Brakke's [28] $m_{n}$ data and multiplied by -250 in order to be visible on the scale of the figure. Error bars are shown for the dot and open square data; where absent, they are smaller than the data point symbols.

methods. We present it in appendix A

We begin by considering an $n$-sided cell with $n$ very large. Cells with very many sides are extremely rare, but if one occurs, then its environment must look as depicted in figure 2, where the $n$-sided cell of a "central" seed at $O$ is surrounded by $n$ strongly elongated first neighbor cells containing seeds $F_{i}$. Independent evidence for such a geometry comes from work by Lauritsen et al. 30, who Monte Carlo simulated a Hamiltonian favoring the appearance of many-sided cells. In figure 2 four different length scales [29] play a role, each proportional to its own characteristic power of $n$ :

(i) The perimeter $\Gamma_{0}$ of the central $n$-sided cell typically runs within an annulus of center $O$, of radius $R_{\mathrm{c}}=(n / 4 \pi \lambda)^{\frac{1}{2}}$, and of width of order 1 . Hence, provided it remains smooth, the perimeter tends for $n \rightarrow \infty$ towards a circle of radius $R_{\mathrm{c}}$. Similarly, the first neighbor seeds $F_{i}$ will be on a circle of radius $2 R_{\mathrm{c}}$.

(ii) Sufficient smoothness of the perimeter $\Gamma_{0}$ in the limit $n \rightarrow \infty$ is guaranteed by the following property: locally the radial coordinates of the 


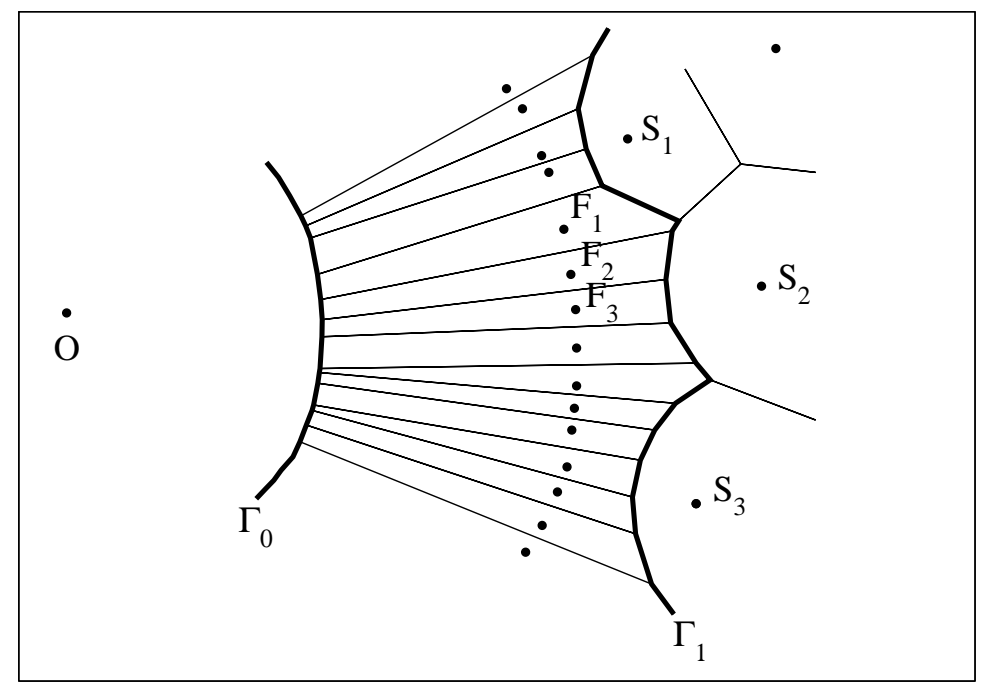

Figure 2: Schematic picture of the environment of an $n$-sided Voronoi cell with $n \approx 100$ around a seed at $O$. The first and second neighbor cells have their seeds at $F_{1}, F_{2}, F_{3}, \ldots$ and at $S_{1}, S_{2}, S_{3}, \ldots$, respectively. All solid line segments separate Voronoi cells. Among these, the heavy solid line $\Gamma_{0}$ is the perimeter of the $n$-sided cell, which is close to circular. The heavy solid line $\Gamma_{1}$ separates the first from the second neighbors. Both $\Gamma_{0}$ and $\Gamma_{1}$ are piecewise linear on a scale $n^{-\frac{1}{2}}$. On the scale of order 1 the incipient piecewise parabolic structure of $\Gamma_{1}$ is discernible. 
individual vertices of $\Gamma_{0}$ have rms deviations of order $n^{-\frac{3}{2}}$ with respect to the locally averaged radial coordinate. A similar statement holds for the curve, not drawn in the figure 2, that links the successive first neighbors.

(iii) The $n$ vertices on the perimeter of the central cell have a line density $\rho_{\text {vert }}=n /\left(2 \pi R_{\mathrm{c}}\right)=(n \lambda / \pi)^{\frac{1}{2}}$. Consequently, the first neighbor seeds $F_{i}$ have a line density $\frac{1}{2} \rho_{\text {vert }}$.

(iv) In the region to the right of the heavy solid line $\Gamma_{1}$, which is occupied by second and further neighbors, the seed density keeps its "background" value $\lambda$, i.e. is of order $n^{0}$ 31.

On this picture we base the following line of arguments. For $n \rightarrow \infty$ the vertex line density $\rho_{\text {vert }}=(n \lambda / \pi)^{\frac{1}{2}}$ tends to infinity. In spite of this the areal density $\lambda$ of the seeds to the right of $\Gamma_{1}$ stays of order $n^{0}$. It follows that the central cell will have $\sim n^{\frac{1}{2}}$ second neighbor cells. Since there are $n$ first neighbors, each second neighbor cell $S_{j}$ must be adjacent to $\sim n^{\frac{1}{2}}$ first neighbor cells $F_{i}$ (where we call the cells by the names of their seeds and where the symbol $\sim$ denotes asymptotic proportionality). Figure 2 shows that under these geometrical constraints each first neighbor $F_{i}$ is most likely to have itself four neighbors, viz. the central $n$-sided cell, a single second neighbor cell, and two other first neighbors, $F_{i-1}$ and $F_{i+1}$.

We now focus on the exceptional $F_{i}$ that have five neighbors due to their being adjacent to two second neighbors $S_{j}$ and $S_{j+1}$. An example is the cell marked $F_{1}$ in figure 2, which is adjacent to both $S_{1}$ and $S_{2}$. Let us denote by $f_{5}$ the fraction of first neighbors that are five-sided. In view of the scaling relations that precede we expect that $f_{5}=c n^{-\frac{1}{2}}+\ldots$, where $c$ is a numerical coefficient and the dots indicate terms of higher order in $n^{-\frac{1}{2}}$. Any six- and higher-sided $F_{i}$ will contribute only to these dot terms. Hence we have

$$
\begin{aligned}
m_{n} & =4\left(1-f_{5}\right)+5 f_{5} \\
& =4+c n^{-\frac{1}{2}}+\ldots
\end{aligned}
$$

It turns out to be possible to determine the constant $c$. To that end we consider the solid line $\Gamma_{1}$ in figure 2, which separates the central seed's first from its second neighbors. In the large- $n$ limit the curve linking the $F_{i}$ becomes a circle which may locally be replaced with a straight line. In figure 3 this straight line is represented by the $x$ axis and the region of space containing the second and further neighbors by the half-plane $y>0$. Seeds are uniformly distributed in the upper half-plane with the background density $\lambda$. Since the first neighbors $F_{i}$ are dense on the $x$ axis, the curve $\Gamma_{1}$ divides the half-plane $y>0$ into a lower part of points closer to the $x$ axis than to any of the seeds, and its complement. Hence the function $y=\Gamma_{1}(x)$ is piecewise parabolic; its incipient parabolic segments are discernable in figure 2. To each cusp of $\Gamma_{1}(x)$ corresponds a five-sided first neighbor cell. Let $\rho_{\text {cusp }}$ be the density on the $x$ axis of the abscissae of the cusps of $\Gamma_{1}(x)$. Then 


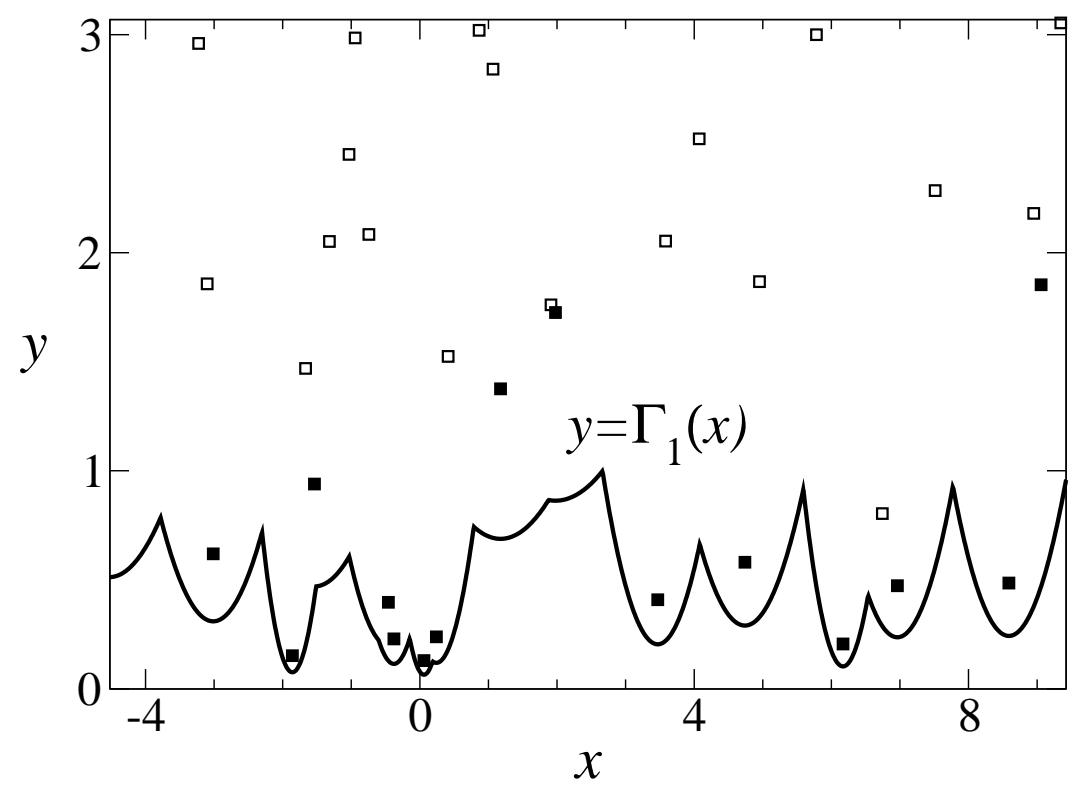

Figure 3: The $x$-axis represents a continuum of first neighbors. The upper halfplane is randomly filled with seeds of uniform density $\lambda$ (here $\lambda=1$ ). The region of the half-plane which is closer to the $x$ axis than to any of the seeds is separated from its complement by the piecewise parabolic curve $y=\Gamma_{1}(x)$. The abscissae of the cusps of $\Gamma_{1}$ are shown in appendix $\mathrm{A}$ to have a density of $\frac{3}{2} \lambda^{\frac{1}{2}}$ on the $x$ axis. Full and open squares represent second and further neighbors, respectively. Note that the horizontal and vertical scales are different. 
it is clear that $f_{5}=2 \rho_{\text {cusp }} / \rho_{\text {vert }}$. The determination of $\rho_{\text {cusp }}$ for given seed density $\lambda$ in the upper half plane is a well-defined problem in statistics. A somewhat lengthy calculation yields $\rho_{\text {cusp }}=\frac{3}{2} \lambda^{\frac{1}{2}}$ (see appendix A), in which the coefficient $\frac{3}{2}$ is essential. Using the expression for $\rho_{\text {vert }}$ found above we therefore have that $f_{5}=2(\pi / n \lambda)^{\frac{1}{2}} \times 3 \lambda^{\frac{1}{2}} / 2=3(\pi / n)^{\frac{1}{2}}$, whence $c=3 \pi^{\frac{1}{2}}$. Substituting this in (2.1) we conclude that for the Random Voronoi Froth $m_{n}$ is given by

$$
m_{n}=4+3 \sqrt{\frac{\pi}{n}}+\ldots, \quad(n \rightarrow \infty)
$$

where the dots stand for higher order terms in $n^{-\frac{1}{2}}$. This exact asymptotic formula for the first-neighbor correlation of Poisson-Voronoi cells is the key result of this work. It represents a new paradigm for the behavior of the cell-cell correlation. We defer all further comments to section 3.

\subsection{Second-neighbor correlation}

It is easy to obtain a corollary involving the correlation between a central cell and its second neighbor cells in the RVF. In a wider context concentric layers of cells around a central one have been the subject of various investigations in recent years 32 . We denote by $K_{n}^{(2)}$ the average number of second neighbors of an $n$-sided cell. The preceding result allows us to determine almost immediately the expression for $K_{n}^{(2)}$, again in the limit of asymptotically large $n$. We appeal once more to figure 2, For $n \rightarrow \infty$ the circle of seeds $F_{i}$ has a circumference $4 \pi R_{\mathrm{c}}=2(n \pi / \lambda)^{\frac{1}{2}}$. The total number $N_{\text {cusp }}$ of cusps of $\Gamma_{1}$ along this circle is equal to $\rho_{\text {cusp }}$ times the circumference, whence $N_{\text {cusp }}=3(\pi n)^{\frac{1}{2}}$. Some caution must be exercised at this point. The number of second-neighbor cells can at most be equal to $N_{\text {cusp }}$; however, it will be slightly smaller. The reason is that the common border between a given second neighbor and the set of first neighbors may consist of more than a single parabolic segment. We therefore have $K_{n}^{(2)}=\eta N_{\text {cusp }}$ where the reduction factor $\eta<1$ is a geometrically defined mathematical constant. The result is that

$$
K_{n}^{(2)}=3 \eta(\pi n)^{\frac{1}{2}}+\ldots, \quad(n \rightarrow \infty) .
$$

We have not seen a way to find a simple analytic expression for $\eta$; from a simulation in which we generated more than 2000 cusps we obtained the estimate

$$
\eta=0.95 \pm 0.01
$$

Equation (2.3) together with (2.4) constitutes the exact asymptotic formula for the total number of second neighbors of a central $n$-sided cell in a Random Voronoi Froth. 


\section{Discussion}

We now discuss the significance of equation (2.2). This equation is first of all a statement of principle: in the RVF (and hence in all theoretical models or experimental systems systems for which it is relevant), as the accuracy and the range of the data increase, the two-cell correlation $m_{n}$ should be seen to follow an $n^{-\frac{1}{2}}$ law and asymptotically approach $m_{\infty}=4$ 33. Equation (2.2) therefore rules out the possibility of the existence of a general proof of Aboav's law for an unrestricted range of $n$. Aboav's law keeps its meaning, however, as a good linear approximation to the available data in the window accessible to simulations or experiments.

\subsection{Comparison to RVF simulation data}

Earnshaw and Robinson [12, following a remark for which they credit Weaire, have emphasized that in order to detect small deviations from Aboav's law it is essential to plot the $m_{n}$ data as a function of $n^{-1}$. We fully concur with them and have plotted in this way in figure 4 the same data that were shown in figure 1. In the new variable $n^{-1}$ Aboav's law remains linear and the negative curvature of the simulation data now appears clearly. In addition we have represented by a solid line in figure 4 the first two terms, $m_{n}=$ $4+3(\pi / n)^{\frac{1}{2}}$, of equation (2.2). We discuss separately two aspects of the theory, viz. its prediction of the curvature effect and its numerical accuracy.

Curvature. - We confront our calculation of the asymptotic behavior of $m_{n}$ with the RVF simulation data. It is visually apparent from figure 4 that the simulation data have a curvature very similar to that of the theoretical curve. Quantitatively, we obtain from (2.2) analytically

$$
-\frac{\left(n m_{n}\right)^{\prime \prime}}{n m_{n}}=\frac{3 \pi^{\frac{1}{2}}}{4 n^{\frac{5}{2}}\left[4+3(\pi / n)^{\frac{1}{2}}\right]},
$$

which ranges from 0.006 to 0.001 in the interval $4 \leq n \leq 8$ and hence is of the same order of magnitude as the value 0.004 found from figure 1 . We conclude that the asymptotic result (2.2) not only explains why the $m_{n}$ data should be curved, but correctly predicts the sign and magnitude of the curvature.

Numerical accuracy. - Equation (2.2) is the beginning of an asymptotic series in powers of $n^{-\frac{1}{2}}$. Although they may, such series are not guaranteed to produce accurate numerical values when the expansion variable is finite. Figures 2 (which is for $n \approx 100$ ) and 3 (for $n=\infty$ ), used in our derivation, refer to seed configurations that are very far away indeed from those observed in either simulations or experiments. Therefore, one might be uninclined a priori to expect of this expansion a high degree of numerical accuracy. However, figure 4 shows the contrary. When compared to the numerical RVF values, our asymptotic result (2.2), limited to its first two terms, is 


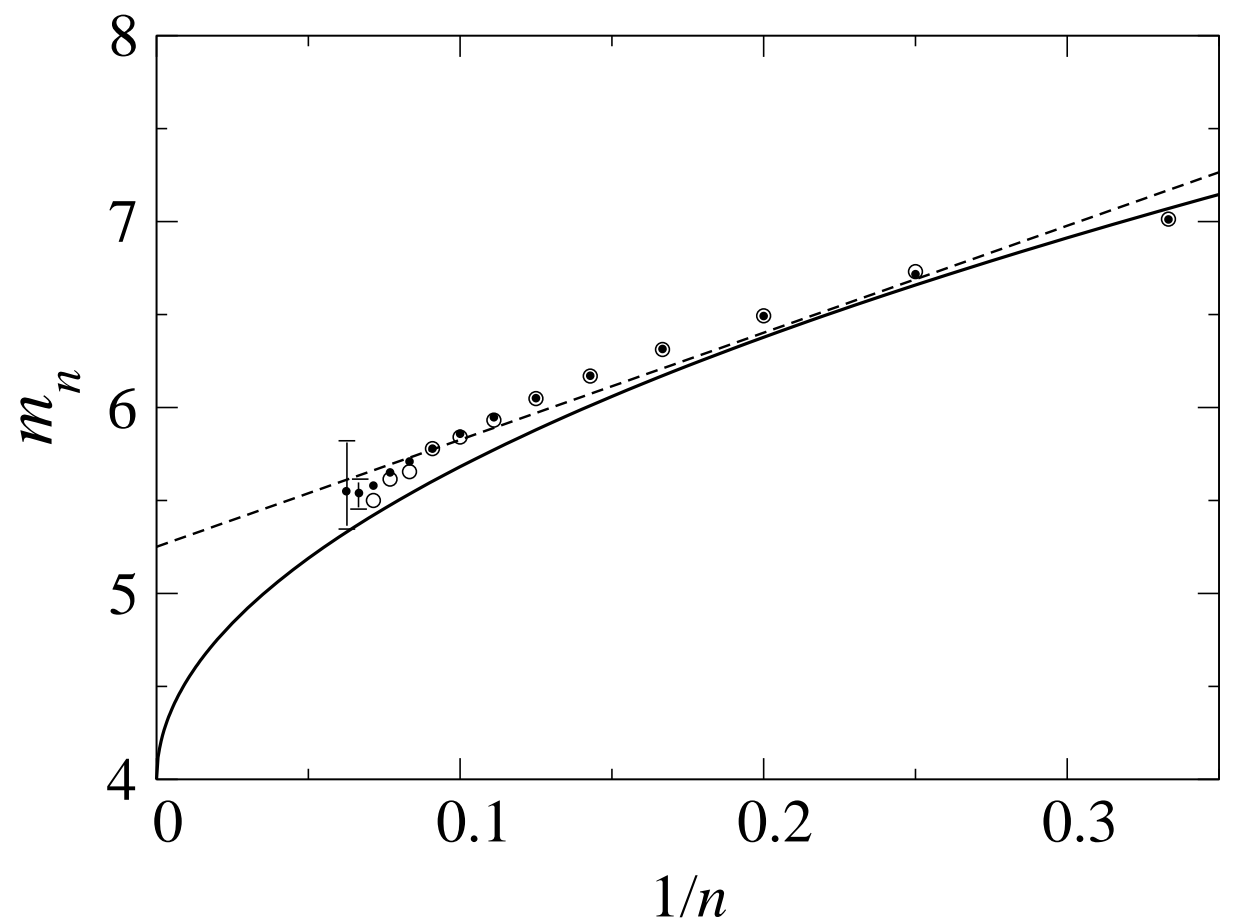

Figure 4: Average sidedness $m_{n}$ of a neighbor of an $n$-sided cell plotted against $n^{-1}$. Open circles and closed dots: same data as in figure 1, but in this plot the curvature appears more clearly. Dashed straight line: Aboav's law $m_{n}=a+b n^{-1}$ with $a$ and $b$ as in figure 1. Solid line: first two terms, $m_{n}=4+3(\pi / n)^{\frac{1}{2}}$, of the exact asymptotic series (2.2). Error bars are shown for the dotted data; where absent, they are smaller than the data points.

quite good: it is only slightly more off than Aboav's linear fit. This gives confidence in the expansion not only as an asymptotic constraint on the $m_{n}$ curve, but also as a practical tool for estimating this and other correlations. We will present further numerical considerations in section 4 .

\subsection{Comparison to diffusion limited colloidal aggrega- tion (DLCA)}

The RVF is an idealized model which appears in the discussion and analysis of various naturally occurring cellular systems [23. We now investigate the relevance of the RVF cell-cell correlations calculated in section 2 for a real physical system, viz. diffusion limited colloidal aggregation (DLCA) in two-dimensional suspensions. In the experiments one monitors the slow aggregation of particles trapped at the air/water interface and initially randomly distributed. In the early stage of this process isolated clusters appear and video images of the system taken after some time $t$ are analyzed in terms 
of the Voronoi cells constructed around the centers of mass of these clusters. Simulation methods developed for such aggregating systems are known 14 to agree well the experimental studies and we will discuss the simulations first.

Recently detailed Brownian dynamics simulations were performed by Fernández-Toledano et al. 34. Their $m_{n}$ versus $1 / n$ data have been represented in our figure 5. The authors fit the $m_{n}$ data by Aboav's linear law (upper thin straight line in figure 5), but their data points depart from linearity for $n>9$ in an even more pronounced way than those of figure 4. Fernández-Toledano et al. attribute these deviations to statistical uncertainties. Instead, we believe that their simulations were accurate enough for them to actually see the curvature predicted by our asymptotic RVF theory, represented in figure 5 by the solid curve. It predicts a curvature of the same order of magnitude as observed in the data. While both Aboav's law and our curve are compatible with the error bars, our theory provides an explanation whereas the linear fit does not. The authors of reference 34 fit their $K_{n}^{(2)}$ data, shown in figure 6, with a linear curve based on Aboav's law plus some additional hypotheses. The data show, however, a slight but clearly distinguishable curvature of the same sign and magnitude as predicted by our law (2.3); hence we believe they have seen this law.

The simulations were prompted by experimental DLCA studies carried out in the nineties by Earnshaw and Robinson, who among several other questions also tested [12, 13] the validity of Aboav's law. Earnshaw and Robinson fitted their experimental $m_{n}$ versus $1 / n$ data by a straight line and concluded [12] that DLCA accords well with the Aboav-Weaire law. We show one of their data sets in our figure 5. It exhibits the downward curvature that we expect. Our comment is again that whereas within the error bars a linear fit is also compatible with the data, only the curved theoretical law (2.2) provides an explanation. The experimental data seem only a small step away from being able to decisively rule out either one or the other. In view of the agreement observed by the authors between experimental and simulation data in many other respects [14, we are confident that DLCA experiments pushed to greater resolution will make the curvature visible.

We finally ask what the physical reason is why RVF theory is applicable to DLCA. We believe the answer is as follows. One may obtain the Voronoi construction for randomly distributed seeds by starting a circular domain growth simultaneously from each seed and having it stop at the contact points of the circular frontiers. It is likely that in the experiments the depletion zones arising around each cluster play exactly the role of these growing circles. 


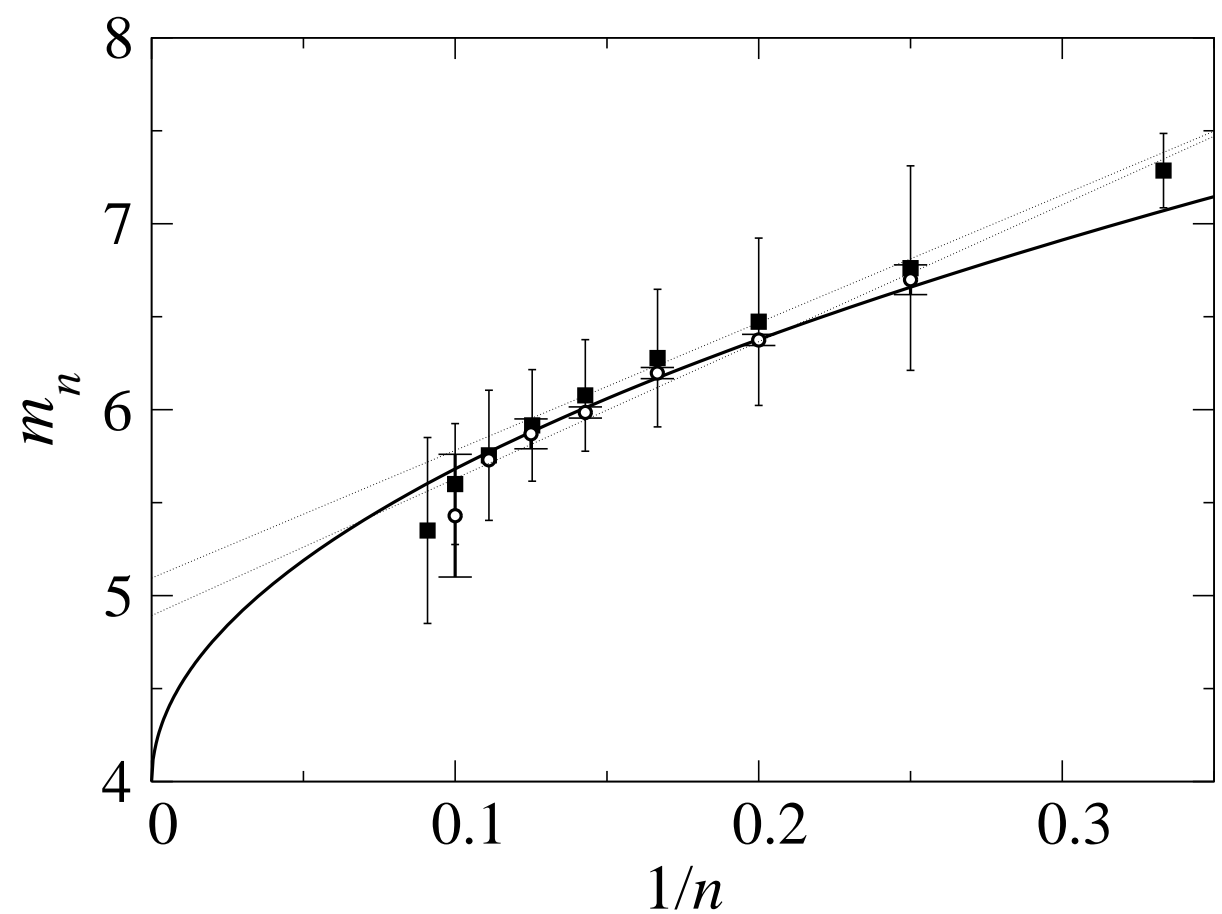

Figure 5: Average sidedness $m_{n}$ of a neighbor of an $n$-sided cell. Full squares: DLCA simulation data by Fernández-Toledano et al. (reference [34, inset of figure 5; the error bars were kindly provided to us by the authors). Open circles: experimental DLCA data by Earnshaw and Robinson (data for $t=60 \mathrm{~min}$ from [12, figure 5). Thin straight lines: Aboav's linear law; the upper one is the fit to the simulation data and the lower one the fit to the experimental data proposed in reference [34] and in reference [12, 13, respectively. Solid curve: first two terms of our theoretical large- $n$ expansion for the Random Voronoi Froth, equation (2.2). 


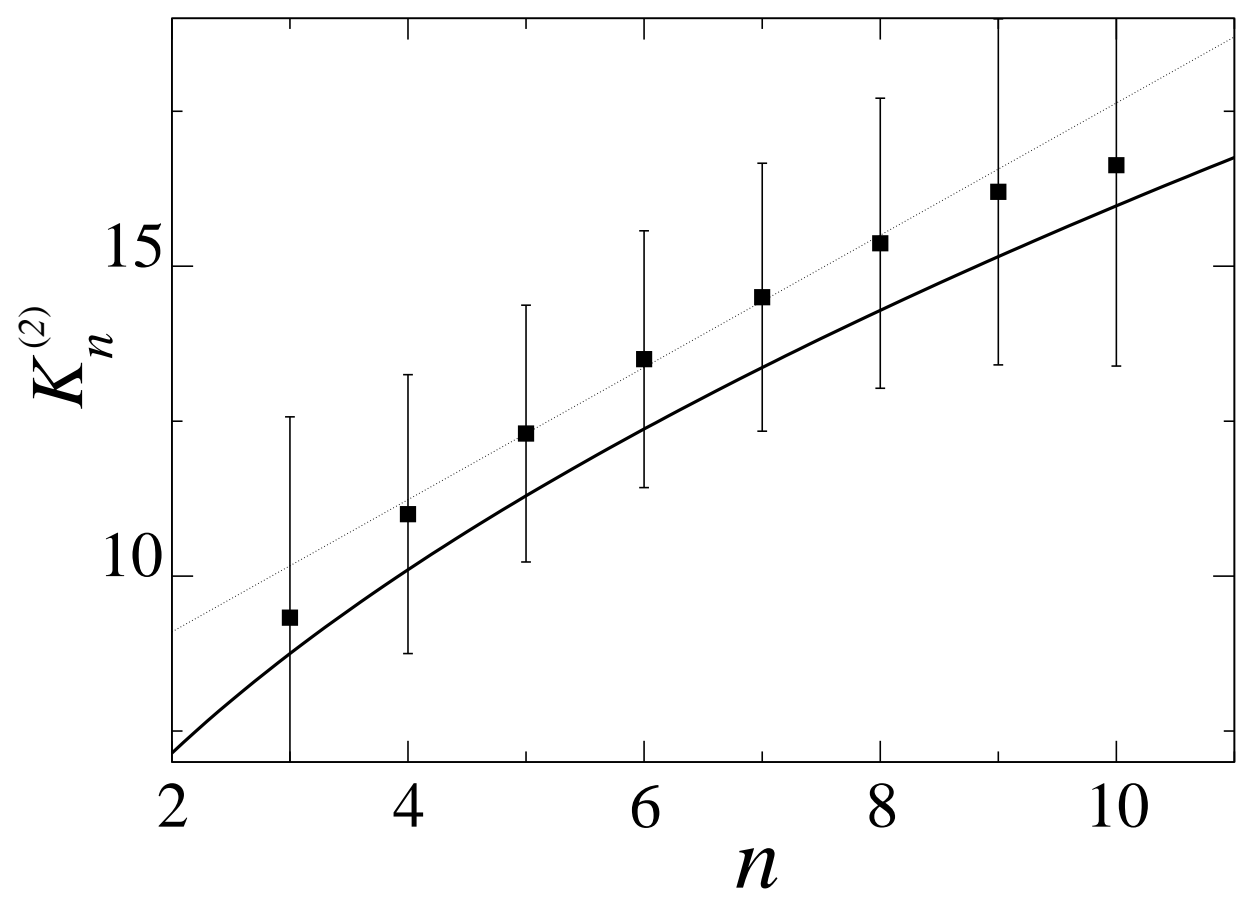

Figure 6: Average number $K_{n}^{(2)}$ of second neighbors of an $n$-sided cell. Full squares: DLCA simulation data by Fernández-Toledano et al. (from [34, figure 7; the error bars were kindly provided to us by the authors). Thin straight line: the linear fit to the data proposed in reference [34. Solid curve: first term of our theoretical large- $n$ expansion for the Random Voronoi Froth, equation (2.3) with $\eta=0.95$. 


\subsection{Comparison to other exact models}

There are very few exact results for the two-cell correlation $m_{n}$ in other geometrical systems. Those that we are aware of all concern model systems artificially constructed to be exactly solvable. An example is the anisotropic "laminated Poisson network" studied by Fortes [35, for which Aboav's law appears to be satisfied exactly (for $n \geq 4$ ) with $a=\frac{4}{3}$ and $b=14$. Several results do exist, however, for topological planar cell models, i.e. models in which only the vertex connectivity is considered but where metric properties such as distances and areas are ignored. To this class belongs very interesting work by Le Caër [36] and Le Caër and Delannay [22]. These authors start from regular planar $z$-coordinated lattices with $z>3$ and by a suitable algorithm construct from it a random topological froth that is three-coordinated just like the RVF and the vast majority of other cellular systems. For one of the simplest models of this kind (the case $z=4$ ) Le Caër [36] finds

$$
m_{n}=\frac{9}{2}+\frac{10}{n}, \quad(n=4,5, \ldots, 8),
$$

which is Aboav's law on a restricted $n$ interval. Other topological models studied by these authors may be treated numerically exactly and, in the general case, show deviations from Aboav's law.

Also in the class of topological models is work by Godrèche et al. [38, who studied an ensemble of diagrams encountered in field theory. For these they found

$$
m_{n}=7+\frac{3}{n}+\frac{9}{n(n+1)},
$$

which while curving upward approaches asymptotically the linear law $n m_{n} \simeq$ $7 n$.

It may well prove useful to classify models of cellular structure according to the decay of their $m_{n}$ by writing generically $m_{n}-m_{\infty} \sim n^{-\alpha}$, where $\alpha$ is a positive exponent. Equations (2.2) and (3.3) then provide examples with $\alpha=\frac{1}{2}$ and $\alpha=1$, respectively. Beyond the RVF studied above it is unknown at present which other theoretical models and experimental systems have $\alpha=\frac{1}{2}$, but for DLCA this certainly seems the likely value. We do not know if it is conceivable that a microscopic geometrical model of cellular structure, as opposed to the topological ones, could have $\alpha=1$. If so, its $n m_{n}$ would obey Aboav's linear law, either exactly for all $n$ or asymptotically for large $n$. Obviously, knowing a system's $\alpha$ value is relevant for the analysis of its data. This value is very likely tied up with the cell formation mechanism, but in an as yet completely unknown way. 


\section{Beyond exact asymptotics: curve fitting}

Equation (2.2) is an exact expansion in powers of $n^{-\frac{1}{2}}$ that we may write as

$$
m_{n}=4+\frac{3 \pi^{\frac{1}{2}}}{n^{\frac{1}{2}}}+\frac{a_{2}}{n}+\frac{a_{3}}{n^{\frac{3}{2}}}+\ldots
$$

The higher order coefficients $a_{2}, a_{3}, \ldots$ are well-defined and it is possible, in principle, to determine them successively. It would certainly be interesting to know a few more of them, but the corresponding calculations are far from straightforward. Moreover, it should be kept in mind that the asymptotic series (4.1) is in all likelihood only asymptotic, which means (see e.g. 39, chapter 1 ) that even though the first few terms may give a good approximation, when adding more and more terms for fixed $n$ one eventually finds that the series does not converge. All we know is that as $n$ grows, (4.1) approaches the exact result ever more closely.

The remainder of this section is in a spirit different from the rest of our work. We include it because of the interest in numerical fits that exists in part of the cellular system community (see, e.g., reference [9]). In the same pragmatic way that led to Aboav's law we now ask if it is possible to fit the numerical $m_{n}$ data by a simple analytic expression that incorporates the first two terms of the asymptotic constraint (2.2). The natural answer is to extend the two explicitly known terms of (2.2) by one or more extra terms with coefficients chosen to fit the data, and to truncate after that. With two extra terms one gets

$$
m_{n}=4+3 \pi^{\frac{1}{2}} n^{-\frac{1}{2}}+A n^{-1}+B n^{-\frac{3}{2}}
$$

where $A$ and $B$ are on the same footing as Aboav's parameters $a$ and $b$. With three extra terms one has

$$
m_{n}=4+3 \pi^{\frac{1}{2}} n^{-\frac{1}{2}}+A n^{-1}+B n^{-\frac{3}{2}}+C n^{-2} .
$$

Here $A, B, C, \ldots$ are not related in any simple way to $a_{1}, a_{2}, a_{3}, \ldots$ of equation (4.1). A relation between the fit parameters arises if one imposes that the fit obey Weaire's sum rule 17

$$
\left\langle n m_{n}\right\rangle=\left\langle n^{2}\right\rangle
$$

in which $\left\langle X_{n}\right\rangle \equiv \sum_{n=3}^{\infty} X_{n} p_{n}$ for any function $X_{n}$ of the sidedness $n$, and $p_{n}$ is the probability that an arbitrarily chosen cell have $n$ sides. Thus, combining (4.4) with Aboav's law (1.2) leads to the well-known relation $b=\mu_{2}+6 a$ where $\mu_{2} \equiv\left\langle n^{2}\right\rangle-\langle n\rangle^{2}$ and one uses that $\langle n\rangle=6$. Imposing Weaire's sum rule makes good sense and we will therefore do the same. After substituting the assumed expressions (4.2) and (4.3) in (4.4) we find

$$
A+B\left\langle n^{-\frac{1}{2}}\right\rangle=12+\mu_{2}-3 \pi^{\frac{1}{2}}\left\langle n^{\frac{1}{2}}\right\rangle .
$$


and

$$
A+B\left\langle n^{-\frac{1}{2}}\right\rangle+C\left\langle n^{-1}\right\rangle=12+\mu_{2}-3 \pi^{\frac{1}{2}}\left\langle n^{\frac{1}{2}}\right\rangle
$$

respectively. The numerically most accurate data for $p_{n}$ and $m_{n}$ available today are those due to Brakke [28]. They lead to $\mu_{2}=1.7807,\left\langle n^{\frac{1}{2}}\right\rangle=2.4344$, $\left\langle n^{-\frac{1}{2}}\right\rangle=0.4160$, and $\left\langle n^{-1}\right\rangle=0.1753$, which are all needed in (4.5) and (4.6).

We fitted both expressions (4.2) and (4.3) by minimizing the weighted maximum deviation from the data, i.e., letting $m_{n}^{\mathrm{B}}$ denote Brakke's numerical values and $\sigma_{n}^{\mathrm{B}}$ their standard deviation, by searching for

$$
\epsilon=\min \max _{3 \leq n \leq 15}\left|m_{n}-m_{n}^{\mathrm{B}}\right| / \sigma_{n}^{\mathrm{B}}
$$

where the minimum is taken over the fit parameters under either constraint (4.5) or (4.6). The results are shown in Table I. As witnessed by the value of $\epsilon$, the two-parameter fit still runs far outside of the standard deviations given by Brakke. The three-parameter fit yields strongly improved results. Comparison of the two- and three-parameter fit shows, in particular, that as more terms are added, there is no sign that the values of $A, B, \ldots$ converge. This also is a strong indication of the merely asymptotic character of the $n^{-\frac{1}{2}}$ expansion.

We emphasize that whereas sections 2 and 3 of our work rest on first principles, the procedure followed in the present section does not. It is hybrid in that it represents the construction of a best fit which has been made to include the first two terms of the exact large- $n$ expansion. There is of course no reason to believe that $m_{n}$ can be represented as a sum of a finite number of powers of $n$, whether it be (4.2), (4.3), or Aboav's law (1.2).

\section{Theory for non-RVF systems}

The RVF serves as a model of reference for general cellular systems much in the same way as an ideal gas does for interacting gases: some of the RVF result will apply to other systems and some will not. There are many factors that may potentially cause an experimental system to depart from RVF-like behavior. E.g., (i) in some cellular structures the 'seeds' represent actual particles or larger physical entities whose mutual interactions cannot be neglected; (ii) a general planar cell structure cannot be derived from a set of point centers by means of the Voronoi construction; and (iii) some systems, like soap froths, are not in equilibrium but rather in a coarsening state and need a dynamical theory in terms of cell transformation processes. We refer to Rivier [23] for an overview.

We now ask the subtler question as to whether the cell-cell correlation $m_{n}$ in any of these non-RVF systems also deviates from Aboav's law by some slight curvature, and if it does, what the asymptotic behavior of its $m_{n}$ is. 


\begin{tabular}{|c|c|c|c|c|c|}
\hline & \multicolumn{2}{|c|}{$\begin{array}{c}\text { Two-parameter fit } \\
\begin{array}{c}A=3.816 \\
B=-7.163\end{array}\end{array}$} & \multicolumn{2}{|c|}{$\begin{array}{l}\text { Three-parameter fit } \\
\begin{array}{c}A=6.279 \\
B=-18.327 \\
C=12.440 \\
\epsilon=7.013\end{array} \\
\end{array}$} & $\begin{array}{l}\text { Simulation data } \\
\text { (Brakke [28] })\end{array}$ \\
\hline$n$ & $m_{n}$ & $m_{n}-m_{n}^{\mathrm{B}}$ & $m_{n}$ & $m_{n}-m_{n}^{\mathrm{B}}$ & $m_{n}^{\mathrm{B}} \pm \sigma_{n}^{\mathrm{B}}$ \\
\hline 3 & 6.9635 & -0.0481 & 7.0183 & 0.0067 & $7.0116 \pm 0.0095$ \\
\hline 4 & 6.7173 & 0.0002 & 6.7152 & -0.0019 & $6.7171 \pm 0.0028$ \\
\hline 5 & 6.5005 & 0.0083 & 6.4923 & 0.0001 & $6.4922 \pm 0.0017$ \\
\hline 6 & 6.31943 & 0.00442 & 6.31593 & 0.00092 & $6.31501 \pm 0.00014$ \\
\hline 7 & 6.16816 & -0.00256 & 6.17114 & 0.00042 & $6.17072 \pm 0.00016$ \\
\hline 8 & 6.04042 & -0.00974 & 6.04932 & -0.00084 & $6.05016 \pm 0.00021$ \\
\hline 9 & 5.93116 & -0.01622 & 5.94497 & -0.00242 & $5.94739 \pm 0.00035$ \\
\hline 10 & 5.83659 & -0.02200 & 5.85429 & -0.00431 & $5.85860 \pm 0.00064$ \\
\hline 11 & 5.7538 & -0.0254 & 5.7746 & -0.0047 & $5.7793 \pm 0.0014$ \\
\hline 12 & 5.6807 & -0.0297 & 5.7038 & -0.0066 & $5.7104 \pm 0.0082$ \\
\hline 13 & 5.6155 & -0.0364 & 5.6404 & -0.0115 & $5.6519 \pm 0.0082$ \\
\hline 14 & 5.557 & -0.0261 & 5.583 & 0.000 & $5.583 \pm 0.023$ \\
\hline 15 & 5.504 & -0.0310 & 5.531 & -0.004 & $5.535 \pm 0.070$ \\
\hline
\end{tabular}

Table 1: Two-parameter fit (4.2) and three-parameter fit (4.3) to Brakke's simulation data 28]. The latter are denoted here as $m_{n}^{\mathrm{B}}$ and their statistical error as $\sigma_{n}^{\mathrm{B}}$. Both fits have been made to satisfy Weaire's sum rule (4.4). 
For certain microscopic models one may hope to be able to find the answer to this question perturbatively starting from the analysis of the present work.

To show how such an approach might proceed we become more speculative. We discuss briefly and heuristically an example from class (ii) above, viz. the Voronoi tessellation associated with a gas of hard core particles of finite diameter $d$. For this system the preceding analysis of the large $n$ limit remains valid as long as $n \lesssim n^{*}$, where the crossover value $n^{*}$ is determined by the condition that the distance between adjacent first neighbors become comparable to the particle diameter. This gives $2 \pi R_{\mathrm{c}} / n^{*} \sim d$ whence, because of $R_{\mathrm{c}}=(n / 4 \pi \lambda)^{\frac{1}{2}}$, it follows that $n^{*} \sim 1 /\left(\lambda d^{2}\right)$, with the $\sim$ sign indicating asymptotic proportionality. For $n \gtrsim n^{*}$ the repulsion between the particles combined with the condition that they be locally aligned along a circle imposes that the radius of the central cell grows as $R_{\mathrm{c}} \sim n d$ and hence that $f_{5}$ must saturate at a value $f_{5}=c_{0} \lambda^{\frac{1}{2}} d$ where $c_{0}$ is an unknown numerical coefficient. The correlation $m_{n}$ can depend only on the two dimensionless variables $n$ and $\lambda d^{2}$. It is reasonable to assume that in the scaling limit $n \rightarrow \infty, \lambda d^{2} \rightarrow 0$ with $n \lambda d^{2}=x$ fixed, $m_{n}$ may be expressed as

$$
m_{n} \simeq 4+\lambda^{\frac{1}{2}} d \mathcal{M}\left(n \lambda d^{2}\right)
$$

in which the scaling function $\mathcal{M}$ must satisfy

$$
\mathcal{M}(\infty)=c_{0}, \quad \mathcal{M}(x) \simeq \frac{3 \pi^{\frac{1}{2}}}{x^{\frac{1}{2}}} \text { for } x \rightarrow 0 .
$$

Hence for hard core particles the limiting value $m_{\infty}$ of (2.2) is changed and (5.2) does not tell us how this new limit is approached for large $n$. However, the $n^{-\frac{1}{2}}$ decay law and the curvature effect that it entails survive in the crossover regime $n \lesssim \lambda d^{2}$, and this is the regime that is encountered first when the experimental or simulational precision increases. Future work will have to deal with this and other instances of deviations from RVF statistics.

\section{Conclusion}

Aboav's linear law (1.2) for the two-cell correlations $m_{n}$ has been known to fail for the Random Voronoi Froth. The exact calculation of this work has explained both qualitatively and quantitatively why it must fail: the exact asymptotic formula for $m_{n}$ exhibits an inverse square root decay with $n$. This large- $n$ behavior represents a new paradigm in the field of planar cellular systems.

In our discussion we have raised the question of whether similar violations occur also in other planar cellular systems, be they theoretical models or experimental realizations. We found that departures from Aboav's law are 
convincingly present in computer simulations of diffusion limited colloidal aggregation (DLCA) performed recently by Fernández-Toledano et al. 34. Since DLCA simulations have been found to be in all respects close to the corresponding experiments [14, it is very likely that DLCA experiments violate Aboav's linear law in the same way. Experiments by Earnshaw and Robinson [12, 13, in spite of error bars that do not unambiguously rule out Aboav's law, provide evidence for the curvature effect. One must expect that future experimental data (or perhaps even reanalysis of existing data), provided they are of high enough precision and/or cover a large enough range in $n$, will reveal the presence of curvature in the $m_{n}$ versus $1 / n$ relation also for certain cellular structures other than DLCA.

The parameters $a$ and $b$ of Aboav's law are useful in that they provide a rough global classification of the behavior of a planar cellular system in the experimentally accessible range of measurement. No first-principle analysis of any geometrical cell model has, however, produced an interpretation and theoretical expressions for these parameters. Empirically, moreover, the precise values of $a$ and $b$ depend on the range of the fit and on how it is performed [36]. Therefore in future high resolution simulations or experiments a fixation on Aboav's law would be misguided and any curvature effect, whenever there is evidence for it, will be worthy of study.

Acknowledgment. - This work was made possible by a six month sabbatical period (CRCT) granted to the author by the French Ministry of Education in the academic year 2004-2005. The author thanks Dr. J.C. FernándezToledano for making his error bar data available to him. He also thanks Dr. K.A. Brakke for correspondence and Dr. N. Rivier for a discussion.

\section{A Appendix}

Equation (2.2) is the central result of this paper. Determining the coefficient of the second term in this equation involves a problem in statistics that we solve in this appendix. We have to prove the following theorem, illustrated by figure 3 .

THEOREM. Let point particles ('seeds') be randomly distributed with uniform density $\lambda$ in the upper half plane $y>0$. Let $\Gamma_{1}(x)$ be the piecewise parabolic curve that separates the upper half plane into a region of points closer to the $x$ axis than to any of the seeds, and its complement. Consider the set of abscissae of the points where the parabolic segments join and where therefore $\Gamma_{1}(x)$ has cusps. Then the density $\rho$ of the abscissae on the $x$ axis is equal to $\rho=\frac{3}{2} \lambda^{\frac{1}{2}}$.

ProOF. The nontrivial part of this theorem is the coefficient $\frac{3}{2}$, since the seed density $\lambda$ may be scaled away; below we keep $\lambda$ only to have at all times 
a check on the dimensionality of the quantities involved in the calculation.

Let $P_{i}=\left(x_{i}, y_{i}\right)$ be the position of the $i$ th seed. The parabola

$$
f_{i}(x)=\frac{y_{i}}{2}\left(1+\frac{\left(x-x_{i}\right)^{2}}{y_{i}^{2}}\right)
$$

separates the upper half plane into a region containing all points closer to the $x$ axis than to $P_{i}$, and its complement. Each parabolic segment of $\Gamma_{1}(x)$ lies on one of the $f_{i}(x)$.

For the considerations that follow we must, as is always implied in statistical mechanics, take a finite system and let eventually its size tend to infinity. We will start with a rectangular box $[-L, L] \times[0, L]$ whose volume we denote by $V=2 L^{2}$ [40]. Let the seeds inside this box be those of indices $i=1,2, \ldots, N$, where $N$ is such that $N / V=\lambda$.

We choose a small $\Delta x$ and define $\rho \Delta x$ as the probability that there is a cusp with abscissa in the interval $[0, \Delta x]$. This is the probability that there exist two parabolas, say $y=f_{j}(x)$ and $y=f_{k}(x)$, that have a point of intersection $P_{j k}=\left(x_{j k}, y_{j k}\right)$ with $x_{j k} \in[0, \Delta x]$ and such that $P_{j k}$ is below all the parabolas $y=f_{\ell}(x)$ with $\ell \neq j, k$. This may be expressed as

$$
\rho \Delta x=\int_{-L}^{L} \prod_{1 \leq i \leq N} \frac{\mathrm{d} x_{i}}{2 L} \int_{0}^{L} \prod_{1 \leq i \leq N} \frac{\mathrm{d} y_{i}}{L} \sum_{1 \leq j<k \leq N} \chi_{j k}(\Delta x) \prod_{\substack{1 \leq \ell \leq N \\ \ell \neq j, k}} \theta_{\ell}\left(y_{j k}\right)
$$

where

$$
\chi_{j k}(\Delta x)= \begin{cases}1 & \text { if } 0 \leq x_{j k} \leq \Delta x \\ 0 & \text { otherwise }\end{cases}
$$

and

$$
\theta_{\ell}\left(y_{j k}\right)= \begin{cases}1 & \text { if } f_{\ell}\left(x_{j k}\right)>y_{j k} \\ 0 & \text { otherwise }\end{cases}
$$

The sum in the integrand in A.2 is on $\frac{1}{2} N(N-1)$ terms that all give identical results so that we may choose $j, k=1,2$. The $N-2$ integrals on $\left(x_{3}, y_{3}\right), \ldots,\left(x_{N}, y_{N}\right)$ may be carried out independently; we have $\theta_{\ell}\left(y_{12}\right)=1$ unless $\left(x_{\ell}, y_{\ell}\right)$ is in the disk of radius $y_{12}$ centered at $\left(x_{12}, y_{12}\right)$. Hence

$$
\int_{-L}^{L} \mathrm{~d} x_{\ell} \int_{0}^{L} \mathrm{~d} y_{\ell} \theta_{\ell}\left(y_{12}\right)=V-\pi y_{12}^{2}
$$

Using this we may rewrite (A.2) as

$$
\rho \Delta x=\frac{N(N-1)}{2 V^{N}} \int_{-L}^{L} \mathrm{~d} x_{1} \int_{-L}^{L} \mathrm{~d} x_{2} \int_{0}^{L} \mathrm{~d} y_{1} \int_{0}^{L} \mathrm{~d} y_{2} \chi_{12}(\Delta x)\left[V-\pi y_{12}^{2}\right]^{N-2} .
$$


In the limit $N, V, L \rightarrow \infty$ with $N / V=N /\left(2 L^{2}\right)=\lambda$ fixed this becomes

$$
\rho \Delta x=\frac{1}{2} \lambda^{2} \int_{-\infty}^{\infty} \mathrm{d} x_{1} \int_{0}^{\infty} \mathrm{d} y_{1} \int_{-\infty}^{\infty} \mathrm{d} x_{2} \int_{0}^{\infty} \mathrm{d} y_{2} \chi_{12}(\Delta x) \exp \left(-\lambda \pi y_{12}^{2}\right) .
$$

The exponential factor in the integrand of (A.7) has the interpretation of the probability that the disk of radius $y_{12}$ around the point of intersection $P_{12}$ contain no seeds. For $i=1,2$ we now transform from the pair of variables $\left(x_{i}, y_{i}\right)$ to the new pair $\left(r_{i}, s_{i}\right)$ defined by

$$
\begin{aligned}
r_{i} & \equiv f_{i}(0)=\frac{y_{i}}{2}\left(1+\frac{x_{i}^{2}}{y_{i}^{2}}\right) \\
s_{i} & \equiv f_{i}^{\prime}(0)=-\frac{x_{i}}{y_{i}}, \quad(i=1,2) .
\end{aligned}
$$

The transformation has the Jacobian

$$
\frac{\partial\left(r_{i}, s_{i}\right)}{\partial\left(x_{i}, y_{i}\right)}=\frac{\left(1+s_{i}^{2}\right)^{2}}{4 r_{i}}, \quad(i=1,2)
$$

In terms of these new variables of integration (A.7) becomes

$$
\begin{aligned}
\rho \Delta x= & 8 \lambda^{2} \int_{0}^{\infty} \mathrm{d} s_{1} \int_{-\infty}^{\infty} \mathrm{d} r_{1} \int_{0}^{\infty} \mathrm{d} s_{2} \\
& \times \int_{-\infty}^{\infty} \mathrm{d} r_{2} \frac{r_{1} r_{2}}{\left(1+s_{1}^{2}\right)^{2}\left(1+s_{2}^{2}\right)^{2}} \chi_{12}(\Delta x) \exp \left(-\lambda \pi y_{12}^{2}\right) .
\end{aligned}
$$

On the interval $0 \leq x \leq \Delta x$ the two parabolas $f_{i}(x)$ may be represented by the expansion

$$
f_{i}(x)=r_{i}+s_{i} x+\mathcal{O}\left(\Delta x^{2}\right), \quad(i=1,2) .
$$

The abscissa $x_{12}$ of their intersection point inside this interval, if there is one, is the solution of $f_{1}\left(x_{12}\right)=f_{2}\left(x_{12}\right)$. Using (A.11) we find

$$
x_{12}=\frac{r_{2}-r_{1}}{s_{2}-s_{1}}+\mathcal{O}\left(\Delta x^{2}\right)
$$

The condition $0 \leq x_{12} \leq \Delta x$ imposed by $\chi_{12}$ in (A.10) can be rewritten as

$$
r_{1} \leq r_{2} \leq y_{1}+\left(s_{1}-s_{2}\right) \Delta x, \quad\left(s_{1}>s_{2}\right)
$$

Because of symmetry we may restrict the $s_{2}$ integration in (A.10) to $s_{2}<s_{1}$ if we compensate by an extra factor 2 . Doing so, interchanging then the $r_{2}$ and 
$s_{2}$ integrals, using condition (A.13), and still observing that $y_{12}=r_{2}+\mathcal{O}(\Delta x)$ we obtain from (A.10)

$$
\begin{aligned}
\rho \Delta x= & 16 \lambda^{2} \int_{0}^{\infty} \mathrm{d} r_{1} \int_{-\infty}^{\infty} \mathrm{d} s_{1} \int_{-\infty}^{s_{1}} \mathrm{~d} s_{2} \\
& \times \int_{r_{1}}^{r_{1}+\left(s_{1}-s_{2}\right) \Delta x} \mathrm{~d} r_{2} \frac{r_{1} r_{2}}{\left(1+s_{1}^{2}\right)^{2}\left(1+s_{2}^{2}\right)^{2}} \exp \left(-\lambda \pi r_{1}^{2}\right),
\end{aligned}
$$

valid up to corrections of order $\Delta x^{2}$. Dividing by $\Delta x$ and performing the $r_{2}$ integral in the limit of $\Delta x \rightarrow 0$ we find

$$
\rho=16 \lambda^{2} I_{r}\left(I_{1}-I_{2}\right)
$$

where

$$
\begin{aligned}
I_{r} & =\frac{1}{2} \int_{-\infty}^{\infty} \mathrm{d} r_{1} r_{1}^{2} \exp \left(-\lambda \pi r_{1}^{2}\right) \\
& =\left(4 \pi \lambda^{\frac{3}{2}}\right)^{-1}
\end{aligned}
$$

and

$$
I_{i}=\int_{-\infty}^{\infty} \mathrm{d} s_{1} \int_{-\infty}^{s_{1}} \mathrm{~d} s_{2} \frac{s_{i}}{\left(1+s_{1}^{2}\right)^{2}\left(1+s_{2}^{2}\right)^{2}}, \quad(i=1,2) .
$$

In the expression for $I_{1}$ we interchange the $s_{1}$ and $s_{2}$ integrals, whereas in the one for $I_{2}$ we interchange the names $s_{1}$ and $s_{2}$. The result is that

$$
\begin{aligned}
I_{1}-I_{2} & =\int_{-\infty}^{\infty} \frac{\mathrm{d} s_{2}}{\left(1+s_{2}^{2}\right)^{2}}\left(\int_{s_{2}}^{\infty}-\int_{-\infty}^{s_{2}}\right) \frac{s_{1} \mathrm{~d} s_{1}}{\left(1+s_{1}^{2}\right)^{2}} \\
& =\int_{-\infty}^{\infty} \frac{\mathrm{d} s_{2}}{\left(1+s_{2}^{2}\right)^{2}}\left[-\frac{1}{2\left(1+s_{1}^{2}\right)}\left(\left.\right|_{s_{2}} ^{\infty}-\left.\right|_{-\infty} ^{s_{2}}\right)\right] \\
& =\int_{-\infty}^{\infty} \frac{\mathrm{d} s_{2}}{\left(1+s_{2}^{2}\right)^{3}} \\
& =\frac{3 \pi}{8} .
\end{aligned}
$$

Upon combining (A.15), (A.16), and (A.18) we find

$$
\begin{aligned}
\rho & =16 \lambda^{2} \times\left(4 \pi \lambda^{\frac{3}{2}}\right)^{-1} \times(3 \pi / 8) \\
& =\frac{3}{2} \lambda^{\frac{1}{2}},
\end{aligned}
$$

which is what we had to prove. 


\section{References}

[1] F.T. Lewis, Anatomical Records 38, 341 (1928); 47, 59 (1930); 50, 235 (1931).

[2] J.C.M. Mombach, R.M.C. de Almeida, and J.R. Iglesias, Phys. Rev. E 47, 3712 (1993).

[3] B. Jeune and D. Barabé, Annals of Botany 82, 577 (1998).

[4] D.A. Aboav, Metallography 3, 383 (1970).

[5] S.J. Mejía-Rosales, R. Gámez-Corrales, B.I. Ivlev, and J. Ruiz-García, Physica A 276, 30 (2000).

[6] P. Cerisier, S. Rahal, and N. Rivier, Phys. Rev. E 54, 5086 (1996).

[7] J.A. Glazier, S.P. Gross, and J. Stavans, Phys. Rev. A 36, 306 (1987); J. Stavans and J.A. Glazier, Phys. Rev. Lett. 62, 1318 (1989).

[8] F. Elias, C. Flament, J.-C. Bacri, O. Cardoso, and F. Graner, Phys. Rev. E 56, 3310 (1997).

[9] A. Okabe, B. Boots, K. Sugihara, and S. N. Chiu, Spatial tessellations: concepts and applications of Voronoi diagrams, second edition (John Wiley \& Sons Ltd., Cichester, 2000).

[10] J. Lemaître, A. Gervois, J.P. Troadec, N. Rivier, M. Ammi, L. Oger, and D. Bideau, Phil. Mag. B 67, 347 (1993).

[11] M. Seul, N.Y. Morgan, and C. Sire, Phys. Rev. Lett. 73, 2284 (1994).

[12] J.C. Earnshaw and D.J. Robinson, Phys. Rev. Lett. 72, 3682 (1994).

[13] J.C. Earnshaw and D.J. Robinson, Physica A 214, 23 (1995).

[14] J.C. Earnshaw, M.B.J. Harrison, and D.J. Robinson, Phys. Rev. E 53, 6155 (1996).

[15] P. Moriarty, M.D.R. Taylor, and M. Brust, Phys. Rev. Lett. 89, 248303 (2002).

[16] K. Zahn, R. Lenke, and G. Maret, Phys. Rev. Lett. 82, 2721 (1999);

R.A. Quinn and J. Goree, Phys. Rev. E 64, 051404 (2001).

[17] D. Weaire, Metallography 7, 157 (1974).

[18] I. Zsoldos and A. Szasz, Computational Materials Science 15, 441 (1999). 
[19] Values outside this range, including negative values of $a$, may nevertheless occur, in particular in artificial computer generated structures. See, e.g., reference 37].

[20] S.F. Edwards and K.D. Pithia, Physica A 205, 577 (1994); Gy. Vincze, I. Zsoldos, and A. Szasz, J. Geometry and Physics 51, 1 (2004).

[21] N. Rivier and A. Lissowski, J. Phys. A 15, L143 (1982); N. Rivier, Phil. Mag. B 52, 795 (1985). M.A. Peshkin, K.J. Strandburg, and N. Rivier, Phys. Rev. Lett. 67, 1803 (1991); C. Sire et M. Seul, J. Phys. I France 5, 97 (1995); B. Dubertret, N. Rivier, and M.A. Peshkin, J. Phys. A 31, 879 (1998).

[22] G. Le Caër and R. Delannay, J. Phys. A 26, 3931 (1993).

[23] N. Rivier in: Disorder and Granular Media, eds. D. Bideau and A. Hansen, Elsevier (1993).

[24] G. Schliecker, Advances in Physics 51, 1319 (2002).

[25] B.N. Boots and D.J. Murdoch, Computers and Geosciences 9, 351 (1983).

[26] G. Le Caër and J. S. Ho, J. Phys. A 23, 3297 (1990).

[27] S. Kumar and S.K. Kurtz, Materials Characterization 31, 55 (1993).

[28] K.A. Brakke, unpublished.

Available on http: //www . susqu . edu/brakke/aux/downloads/200.pdf.

[29] H.J. Hilhorst, J. Stat. Mech. L02003 (2005); H.J. Hilhorst, J. Stat. Mech. P09005 (2005).

[30] K.B. Lauritsen, C. Moukarzel, and H.J. Herrmann, J. Phys. I France 3, 1941 (1993).

[31] Figure 2 is only schematic in that a faithful representation of all proportions would lead to an about twice higher density of the second and further neighbors to the right of $\Gamma_{1}$; as it is, the picture better brings out the emergence of the piecewise parabolic segments of $\Gamma_{1}$.

[32] T. Aste, D. Boosé, and N. Rivier, Phys. Rev. E 53, 6181 (1996); T. Aste, K.Y. Szeto, and W.Y. Tam, Phys. Rev. E 54, 5482 (1996); K.Y. Szeto, T. Aste, and W.Y. Tam, Phys. Rev. E 58, 2656 (1998).

[33] This is in agreement with the inequality $m_{n}>4$ for all $n$, derived by Le Caër and Delannay [22]. 
[34] J.C. Fernández-Toledano, A. Moncha-Jordá, F. Martínez-López, A.E. González, and R. Hidalgo-Álvarez, Phys. Rev. E 71, 041401 (2005).

[35] M.A. Fortes, J. Phys. A 28, 1055 (1995).

[36] This dependence was stressed, in particular, by G. Le Caër, J. Phys. A 24, 1307 (1991); 24, 2677 (1991); 24, 4655 (1991). In reference [22] a proposal is made to come to a unique definition (called $a_{w}$ there) of the parameter $a$, valid also when $n m_{n}$ is not linear. The connection of this parameter to the second moment of the $n$ distribution was studied in reference [37.

[37] R. Delannay and G. Le Caër, Phys. Rev. Lett. 73, 1553 (1994).

[38] C. Godrèche, I. Kostov, and I. Yekutieli, Phys. Rev. Lett. 69, 2674 (1992).

[39] N. Bleistein and R.A. Handelsman, Asymptotic Expansions of Integrals, Dover Publications, Inc., New York (1986).

[40] More generally one might take a box $[-L, L] \times\left[0, M_{L}\right]$ where it suffices that $\lim _{L \rightarrow \infty} M_{L}=\infty$. 